\title{
The intermediate nebular phase of SN 2014J: onset of clumping as the source of recombination
}

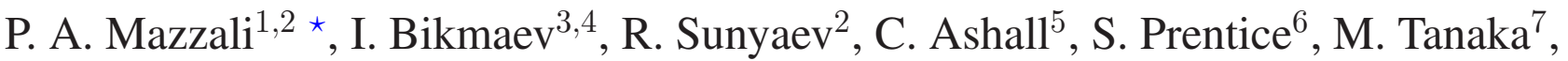 \\ E. Irtuganov ${ }^{3,4}$, S. Melnikov ${ }^{3,4}$, R. Zhuchkov ${ }^{3,4}$ \\ ${ }^{1}$ Astrophysics Research Institute, Liverpool John Moores University, IC2, Liverpool Science Park, 146 Brownlow Hill, Liverpool L3 5RF, UK \\ ${ }^{2}$ Max-Planck-Institut für Astrophysik, Karl-Schwarzschild Str. 1, D-85748 Garching, Germany \\ ${ }^{3}$ Department of Astronomy and Satellite Geodesy, Kazan Federal University, Kremlevskaya Str, 18, Kazan, 420008, Russia \\ ${ }^{4}$ Academy of Sciences of the Republic of Tatarstan, Bauman Str., 20, Kazan, 420111, Russia \\ ${ }^{5}$ Florida State University, Tallahassee, FL, USA \\ ${ }^{6}$ Trinity College Dublin, Ireland \\ ${ }^{7}$ Tohoku Univ., Sendai, Japan \\ Accepted ... Received ...; in original form ...
}

\begin{abstract}
At the age of about 1 year, the spectra of most Type Ia supernovae are dominated by strong forbidden nebular emission lines of Fe II and Fe III. Later observations (at about 2 years) of the nearby SN 2011fe showed an unexpected shift of ionization to Fe I and Fe II. Spectra of the very nearby SN Ia 2014J at an intermediate phase ( $1-1.5$ years) that are presented here show a progressive decline of $\mathrm{Fe}$ III emission, while $\mathrm{Fe}$ I is not yet strong. The decrease in ionization can be explained if the degree of clumping in the ejecta increases significantly at $\sim 1.5$ years, at least in the Fe-dominated zone. Models suggest that clumps remain coherent after about one year, behaving like shrapnel. The high density in the clumps, combined with the decreasing heating rate, would then cause recombination. These data may witness the phase of transition from relatively smooth ejecta to the very clumpy morphology that is typical of SN remnants. The origin of the increased clumping may be the development of local magnetic fields.
\end{abstract}

Key words: supernovae: general - supernovae: individual (SN 2014J) - techniques: spectroscopic - radiative transfer

\section{INTRODUCTION}

Many issues remain unclear about Type Ia supernovae (SNe Ia), although there is agreement that they are the thermonuclear explosion of carbon-oxygen (CO) white dwarfs (WD), and that most of them produce a large amount of radioactive ${ }^{56} \mathrm{Ni}$. A relation between ${ }^{56} \mathrm{Ni}$ mass, luminosity and light curve shape was discovered (Phillips 1993) and explained in terms of the behaviour of the opacity (Pinto \& Eastman 2000; Mazzali et al. 2001a, 2007a). This relation has been applied so that SNe Ia can be used as standardizable candles, leading to the discovery of the accelerated expansion of the Universe (Riess et al. 1998; Perlmutter et al. 1999).

Many different progenitor scenarios have been proposed for SNe Ia. A short list includes: i) the single-degenerate (SD) scenario, where a WD accretes matter from a non-electron degenerate donor companion in a binary system (e.g., Whelan \& Iben 1973; Nomoto et al. 1984); ii) the double-degenerate scenario (DD), which consists of two WDs in a binary system that eventually merge (Webbink 1984; Iben \& Tutukov 1984); iii) the "core-

^ E-mail: P.Mazzali@ljmu.ac.uk degenerate" scenario, where a WD is engulfed in a common envelope by the outer layers of a binary companion when this expands to become an asymptotic giant branch (AGB) star until the WD finally merges with the degenerate core of the AGB star (e.g., Livio \& Riess 2003; Kashi \& Soker 2011), and iv) the explosion triggered by the head-on collision of two WDs, either in a dense environment such as a cluster (Rosswog et al. 2009) in a triple system where the third member is a non-electron-degenerate star, which focuses the orbits of the two WDs (Kushnir et al. 2013). For a recent review of all scenarios see Livio \& Mazzali (2018).

Additionally, within each progenitor system a variety of different explosion mechanisms have been proposed to occur. When a WD approaches the Chandrasekhar mass $(\mathrm{M}(\mathrm{Ch}))$ through accretion from a companion star, compressional heating triggers a thermonuclear runaway in the centre. The flame can propagate at both sub- and super-sonic speeds, leading to different nucleosynthesis and energy yields. In this scenario the companion can be a degenerate or a non-degenerate star (e.g., Iwamoto et al. 1999; Piersanti et al. 2003). In sub-M(Ch) explosions an accreted surface He layer can ignite and cause a shock wave on the surface of the WD. If the shock propagates into the WD it can produce a central 
detonation. Once again this mechanism can occur in both the SD and DD scenarios, through accretion from a He star or another WD with a He layer (Livne \& Arnett 1995; Shen \& Moore 2014).

Although supporters of the different scenarios often claim that their favourite model can explain all $\mathrm{SNeIa}$, it is not unlikely that different, physically plausible channels to a SN Ia actually co-exist. While models of the early-time properties of SNe Ia do not always lead to conclusive results about the specific progenitor/explosion channel (e.g., Röpke et al. 2012), clearer evidence for the existence of multiple channels comes from nebular spectroscopy (e.g., Mazzali et al. 2007a, 2015, 2011, 2018; Mazzali \& Hachinger 2012).

In the late, nebular phase, when the inner parts of the ejecta are visible, distinguishing among different explosion scenarios based on their expected imprint is somewhat easier. This is because the central density, and hence the total mass of the exploding WD directly affects the nucleosynthesis and the density distribution in the inner-most regions of the ejecta, and different models tend to exhibit the largest differences in the inner layers.

In addition to outstanding questions about the nature of the progenitor system and the mode of explosion, the link between the SNe Ia we observe as luminous transient sources and the SN remnants (SNR) they produce also offers interesting puzzles. SN Ia remnants are thought to be quite spherical and homogeneous, which should reflect the properties of the explosion and testify to the lack of significant circumstellar medium (CSM) to shape the remnant at later stages (Patnaude et al. 2017). While this is in line with expectations for progenitor evolution (although in some scenarios, such as WD collisions and mergers, the ejecta may not be expected to be spherically symmetric, e.g., Bulla et al. 2016), those SNRs that are identified as most likely being the result of a SN Ia explosion are characterised by significant clumping. Ferrand et al. (2019) argue that clumps can be developed by Rayleigh-Taylor instabilities, over a timescale of $\sim 100 \mathrm{yr}$. However, the presence of clumping in the first few years after the explosion is not suggested by either the explosion models or by observations in the early phases.

The period in a SN intermediate between light curve peak and the SNR is the so-called nebular phase. This phase is entered when the SN ejecta start to become optically thin, some six months after the $\mathrm{SN}$ explosion, and continues as long as collisional excitation and ionization determine the state of the gas and before impact on any CSM lights up the nebula in a reverse shock and shapes the SNR if the CSM is not spherically symmetric. Because SNe Ia have only been observed in external galaxies in the modern era, rarely has it been possible to follow them in the nebular phase much beyond one year, as they quickly become too faint to be observed spectroscopically.

On the other hand, most well-studied SN remnants are in the Milky Way or the Magellanic Clouds, where they are close enough to be observed. Typical SNRs have ages of 1000 years or more. Therefore, the transition from SN to SNR is practically unexplored. The occurrence in recent years of two SNe Ia in nearby galaxies offers an opportunity to explore more advanced stages of the nebular phase than were previously known. SN 2011fe, a normal SN Ia (Nugent et al. 2011), was monitored in the early nebular phase (up to one year after explosion). Its spectrum showed strong emission lines of both [Fe II] and [Fe III]. The spectra were shown to be consistent with a Chanderasekhar-mass explosion (Mazzali et al. 2015). At more advanced epochs the light curve showed the onset of different radioactivity (in particular ${ }^{57} \mathrm{Fe}$, Taubenberger et al. 2015), as expected from nucleosynthesis calculations. Surprisingly, however, SN 2011fe displayed a shift in ionization, with spectra taken 593 and 981 days after the explosion still showing [Fe II] lines but not [Fe III] lines, which seemed to have been replaced by [Fe I] lines (Graham et al. 2015). A noisy optical spectrum obtained 481 days after explosion (Zhang et al. 2016) appears to show the two strong [Fe II] and [Fe III] emissions near 4800 and $5200 \AA$, but also an emission near $4500 \AA$, which Zhang et al. (2016) identify as [Fe II] but which, based on its wavelength, may also be consistent with the $[\mathrm{Fe} \mathrm{I}]$ emission that is seen at very late times in SN 2011fe. Although it would be unusual for three different ionization stages to be strong at the same time, this is the only evidence of how the transition from [Fe III]- to [Fe I]-dominated spectra in SN 2011fe may have occurred. While some degree of cooling could be expected as the SN ejecta age and expand, the degree of recombination that has been observed in SN2011fe was not predicted by models, which favour a freezout in ionization as the density decreases (Fransson \& Jerkstrand 2015).

Another very nearby, recent SN Ia is SN 2014J, which exploded in M82 and was the closest SN Ia since 1972E. Several papers have focussed on the early-time properties and the unusual extinction of SN 2014J (e.g., Foley et al. 2014; Amanullah et al. 2014; Ashall et al. 2014). Nebular spectra of SN 2014J have been presented in a few papers. Srivastav et al. (2016) presented two spectra taken at epochs $\approx 288$ and 370 days after explosion. They noticed the weakness of the [Fe III]-dominated emission relative to other SNe Ia and interpreted this as a signature of clumping in the ejecta following Mazzali et al. (2001b). Two papers have dealt specifically with late-time, near-infrared (NIR) spectra. Diamond et al. (2018) conclude that SN 2014J was consistent with a $\mathrm{M}(\mathrm{Ch})$ progenitor, which exploded as a delayed detonation with an off-centre ignition. Dhawan et al. (2018) claim to have observed a [Ni II] $1.939 \mu \mathrm{m}$ feature, consistent with high density central burning, which again would be consistent with a $\mathrm{M}(\mathrm{Ch})$ progenitor. Here we present late-time optical spectra of SN 2014J that span epochs from 10 to 14 months after explosion. The spectra do show a progressive decrease of [Fe III] emission, although [Fe I] is not emerging yet, and thus cover the initial part of the ionization shift. Modelling the spectra we obtain an approximate description of the properties of the ejecta and of their changes during the period monitored. These changes lead to recombination in the ejecta and the progressive fading of [Fe III] emission.

\section{SPECTROSCOPIC OBSERVATIONS, DATA SUBTRACTION AND CALIBRATION}

Observations of SN 2014J were performed on the 1.5-m RussianTurkish telescope RTT-150 (Aslan et al. 2001) using time allocated to Kazan Federal University. The low-resolution spectroscopic mode of the TFOSC instrument was used. A log of observations is given in Table 1. A prism in combination with a 100 micron (1.8 arcsec) entrance slit and a 2048 × 2048 pixels nitrogen-cooled CCD provide a spectral resolution of $15 \AA$ in the wavelength range 4000-9000 A. The spectra were reduced using a modified version of the DECH software package ${ }^{1}$. The wavelength scale was calibrated using the comparison spectra of an Fe-Ar lamp. To calibrate the fluxes in the spectra, the spectrophotometric standard stars BD+75d 325 and Feige 34 were observed.

The wavelength calibration accuracy is $\approx 0.1 \AA(\approx 5 \mathrm{~km}$

\footnotetext{
1 www.gazinur.com
} 
Table 1. Log of observations for SN2014J with RTT-150

\begin{tabular}{lccc} 
Date & JD & Days after $B$ maximum & Total Exposure per night (s) \\
\hline 2014 Nov 11 & 2456973 & 283 & 4500 \\
2014 Dec 4 & 2546996 & 306 & 5400 \\
2014 Dec 14 & 2457006 & 316 & 2700 \\
\hline Average & 2456993 & 303 & Total exposure 12600 sec \\
\hline \hline 2015 Jan 21 & 2457044 & 354 & 9000 \\
\hline \hline 2015 Mar 24 & 2457106 & 416 & 9000 \\
2015 Mar 25 & 2457107 & 417 & 5400 \\
\hline Average & 2457106 & 416 & Total exposure 14400 sec \\
\hline
\end{tabular}

$\mathrm{s}^{-1}$ ). An additional uncertainty of $\approx 50 \mathrm{~km} \mathrm{~s}^{-1}$ was caused by the motion of the stellar image perpendicular to the spectrograph entrance slit during the exposure. To increase the signal-to-noise ratio in the spectra of SN 2014J at a late nebular phase, the spectra taken on November 11, December 4 and 14, 2014, were averaged (the average spectrum corresponds to day 303 after maximum brightness), as were the spectra taken on March 24 and 25, 2015, (the average spectrum corresponds to day 416 after maximum brightness).

The observed spectrum of SN 2014J during the late nebular phase is contaminated by underlying host-galaxy light. In order to correct for this, a spectrum of the host at the position of the $\mathrm{SN}$ is required at a time when the $\mathrm{SN}$ light makes a negligible contribution to the total observed flux. A single $800 \mathrm{~s}$ spectrum of M82 at the coordinates of SN 2014J was therefore obtained on 2019 Jan 14.03 (MJD 58497.03) using the SPectrograph for the Rapid Acquisition of Transients (SPRAT; Piascik et al. 2014), installed on the $2 \mathrm{~m}$ Liverpool Telescope (LT; Steele et al. 2004). SPRAT is a low resolution, high throughput spectrograph which covers a wavelength range of $4000-8000 \AA$ and reaches a binned resolution of $\mathrm{R}=350$ at the centre of the spectrum for a slit width of 1.8 arcsec. The late epoch of this exposure ensured that the $\mathrm{SN}$ had faded from view. The data were initially reduced through the standard SPRAT pipeline, which applies corrections for bias, dark, and flat-field. The object spectrum was then extracted, and calibrated in wavelength and flux using a custom PYRAF pipeline. The extracted spectrum was left unbinned, giving a resolution of $\mathrm{R}=634(\approx 9 \AA)$ at $6000 \AA$. A final flux correction, derived from the standard star used to define the sensitivity function, was applied to the output spectrum to correct for telluric absorption.

The spectra of SN 2014J were calibrated in flux with respect to the Hubble Space Telescope (HST) photometry published by Yang et al. (2018). Because only 3 photometric HST bands (F475W, F606W, and F775W) are available, we assumed that the spectra had the correct shape after reduction and proceeded to calibrate them in flux by correcting them using a constant to ensure that they matched the observed photometry.

The spectra were then corrected for extinction and reduced to restframe. For the Milky Way we used $E(B-V)=0.05 \mathrm{mag}$, $R_{V}=3.1$, and for the host galaxy $E(B-V)=1.2 \mathrm{mag}$, $R_{V}=1.38$ (Ashall et al. 2014). This is consistent with the extinction values found in (Foley et al. 2014; Amanullah et al. 2014). The spectral sequence, after correction for extinction and removal of the host galaxy spectrum, is shown in Figure 1.

As Fig. 1 shows, over the period sampled by our data the spectrum of SN 2014J showed a significant change in degree of ionization. The first two epoch were dominated by blends of [Fe III] and
[Fe II] emission lines, as is typical of SNe Ia in the classical nebular phase (up to about one year). At the epoch of the third spectrum however, although the main emission features remained the same as at earlier epochs, the ratio of the two strongest Fe emission changed. While at earlier epochs the [Fe III]-dominated emission near $4700 \AA$ was significantly stronger than the [Fe II]-dominated one near $5200 \AA$, at day 435 the ratio of these two features is reversed, with the $[\mathrm{Fe} \mathrm{II}]$-dominated one now the dominant emission. This may be seen as the beginning of a transition similar to that which occurred in SN2011fe, which showed strong [Fe I] emission at an epoch of $\sim 3$ years after explosion. [Fe I] emission is not observed in the available spectra of SN 2014J, but it may be expected that it could appear, or even become dominant, at later times. Thus motivated, we undetook an analysis of the spectra of SN 2014J using our SN nebular code to explore what could lead to the observed evolution of the spectrum.

\section{THE SN NEBULAR CODE}

We computed synthetic spectra using our non-local thermodynamic (NLTE) nebular emission code for supernovae. The code is built based on the assumptions set out by Axelrod (1980). The gas in the SN nebula is assumed to be heated by collisions with the high-energy particles generated in the thermalization process of the gamma-rays and positrons emitted in the decay chain ${ }^{56} \mathrm{Ni} \rightarrow{ }^{56} \mathrm{Co}$ $\rightarrow{ }^{56} \mathrm{Fe}$, and it cools via the emission of (mostly) forbidden lines. Some strong permitted transitions are also considered.

The deposition of gamma-rays and positrons is computed using a Montecarlo method, as outlined in Cappellaro et al. (1997) and Mazzali et al. (2001a). Constant opacities are used of $\kappa_{\gamma}=$ $0.027 \mathrm{~cm}^{2} \mathrm{~g}^{-1}$ and $\kappa_{e^{+}}=7 \mathrm{~cm}^{2} \mathrm{~g}^{-1}$. The large difference in their respective opacities means that at very late times positrons make the dominant contribution to energy deposition, although they carry only $\approx 3.6 \%$ of the total emitted radioactive energy.

After computing the energy deposition, the ionization and the NLTE thermal balance in the nebula are solved following the prescriptions outlined by, e.g., Ruiz-Lapuente \& Lucy (1992). Ionization is assumed to be provided exclusively by impact with the high-energy particles produced by the deposition of gammarays and positrons. Photoionization is assumed to be negligible (Kozma \& Fransson 1998). Ionization balance is achieved by equating the rate of impact ionization with the recombination rate for each ion. Within each ion, level populations are computed solving the rate equations for each level under the assumption of thermal balance, i.e., equating the non-thermal heating rate and the rate of cooling via line emission. The assumption is made that the 


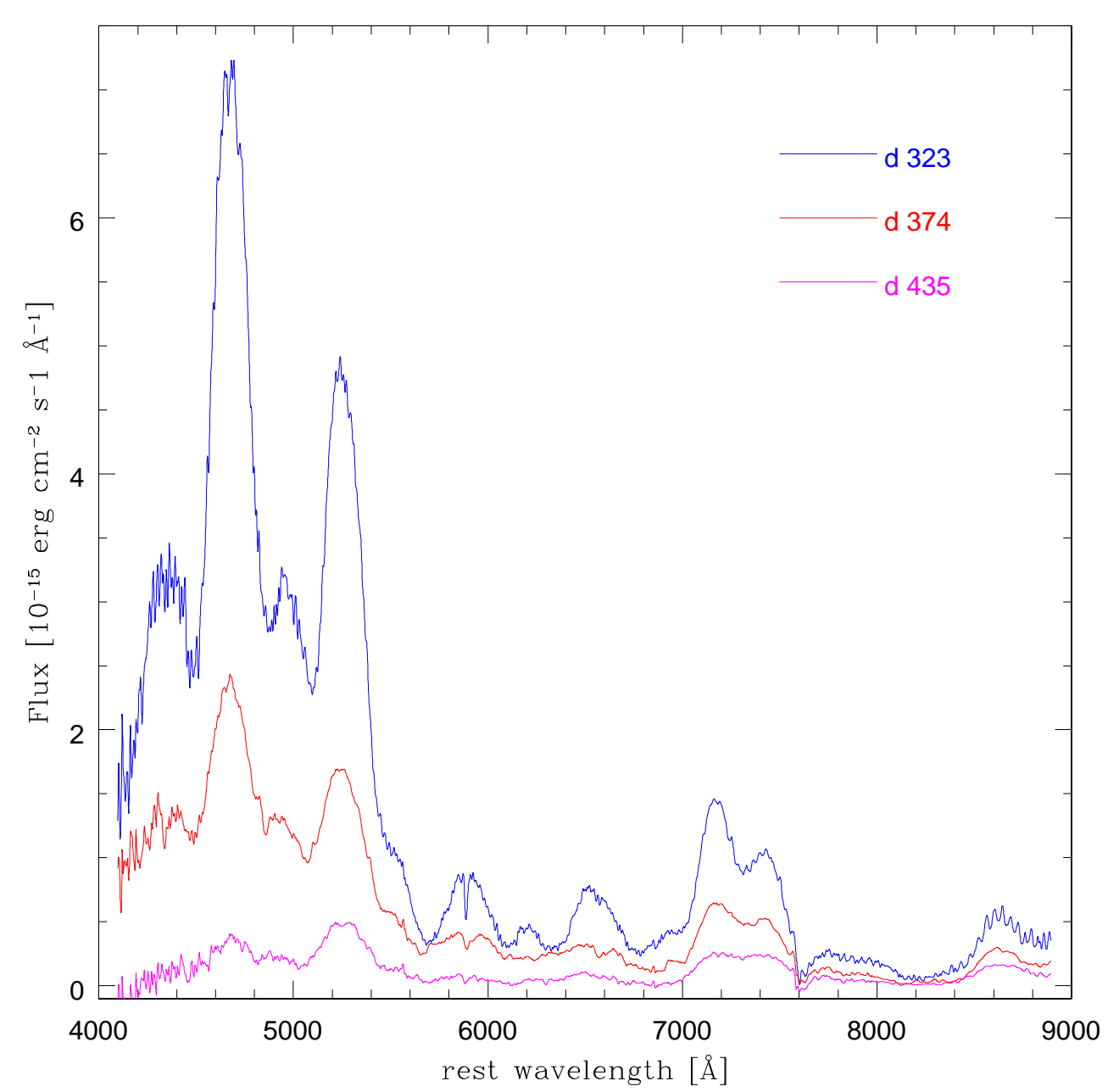

Figure 1. The three nebular spectra of SN 2014J used in this work, in their original flux after correcting for reddening. The spectral sequence highlights the decline of the emission near $4700 \AA$ relative to other emission features.

nebula is optically thin, and radiation transport in not performed. The emissivity in the various lines is used to compute the emerging spectrum.

The code can be run as a simple one-zone code, in which case the line profile is a parabola with characteristic width determined by the speed of expansion of the outer boundary of the nebula (which is input to the code). Alternatively, a stratified version can use a one-dimensional explosion model (i.e., a radially dependent density/abundance profile), in which case energy deposition, ionization balance, level populations and emissivity are computed for each shell. Each shell then contributes its own emissivity. Line profiles are built by summing truncated parabolae bounded by each shell's inner and outer boundary velocity. In both cases, the effects of line blending are automatically taken into account in the computation of the emitted spectrum.

Our code has been used for a number of SNe Ia (e.g., Mazzali \& Hachinger 2012; Mazzali et al. 2011) and Ib/c (e.g., Mazzali et al. 2007b), and it can yield a description of the inner layers of the SN ejecta. When used in combination with a description of the outer layers, which can be obtained through a study of the early spectra, the mapping of the inner ejecta that results from nebular studies can be used to obtain a full description of the density and abundance distribution in a SN (e.g., Mazzali et al. 2015; Ashall et al. 2016, 2018).

One aspect our code attempts to treat is clumping of the ejecta. The simple assumption is made that all ejecta reside in clumps, which occupy a fraction of the total volume, as defined by a filling factor. The density in the clumps is then increased following the decrease in volume, and the composition of the clumps is set to be the same as that of the radial ejecta shell they are in, i.e., all clumps are homogeneously and microscopically mixed. The clumps are still assumed to be in thermal equilibrium. These are of course major simplifications, but in the inner layers of a SN Ia only few elements are present, so it is not unreasonable. Strong clumping has been shown to be very useful in order to reproduce the low ionization seen in late-time spectra of SNe Ic (Mazzali et al. 2001b, e.g., ), where it may be thought of as a proxy for the non-spherically symmetric distribution of the ejecta. Clumping is used later in this paper to improve our synthetic spectra, especially in the final epoch. 


\section{SPECTRAL MODELS AND THE EVOLVING PROPERTIES OF SN 2014J}

We started our analysis by deriving some basic properties of the emitting nebula using the one-zone version of our code. The flux progressively decreases with time, as is expected for a spectrum that is powered by the decay of ${ }^{56} \mathrm{Co}$, but the width of the emission lines decreases only marginally, suggesting that over the time monitored the nebular emitting region remains roughly constant. This confirms the results of Mazzali et al. (1998).

From simple 1-zone fits to the spectra we estimate a typical expansion velocity of the emitting nebula of $8500 \pm 500 \mathrm{~km} \mathrm{~s}^{-1}$. This places SN 2014J among relatively luminous SNe Ia, according to the scheme of Mazzali et al. (2007a). For normal SNe Ia this emission line width corresponds to decline rates of $\Delta(m)_{15} \sim$ $(1.1-1.3)$ mags. This is reasonably consistent with the observed value for SN 2014J, which had an extinction-corrected decline rate $\Delta(m)_{15}=1.08$ mags. SNe Ia with this range of decline rates typically have a ${ }^{56} \mathrm{Ni}$ mass of $\sim(0.4-0.6) \mathrm{M}_{\odot}$ (Mazzali et al. 2007a).

A much more accurate description of the properties of the young SN nebula can be obtained using the stratified version of the code in combination with an explosion model. As an example, using this approach we have been able to show that the peculiar behaviour of the nebular specta of some underluminous SNe Ia, namely the unexpected increase in ionization at late times, can be explained if the innermost ejecta contain little mass, and in particular no stable Fe, such as predicted by sub-Chandrasekhar mass models as a consequence of the low central density of the progenitor white dwarfs (Mazzali et al. 2011; Mazzali \& Hachinger 2012). The low density suppresses recombination, and the absence of stable Fe reduces the cooling rate, leading to the observed effects. In the case of SN 2014J the behaviour is the opposite.

The question then arises of which explosion model we should select. A study of the properties of SN 2014J based on the early spectral evolution and light curve was performed by Ashall et al. (2014). They fitted a series of spectra and concluded that SN 2014J was consistent with a normal, Chandrasekhar-mass SN Ia, with kinetic energy $E_{K} \sim 1.210^{51} \mathrm{erg}$. The classical, albeit artificially fast deflagration model W7 (Nomoto et al. 1984) proved perfectly adequate to reproduce the early spectra, requiring only minor modifications to the abundances. That work could only probe the outer ejecta, down to a velocity of $\sim 8500 \mathrm{~km} \mathrm{~s}^{-1}$.

Here, therefore, we follow Ashall et al. (2014) and adopt the density structure of W7 to sample the lowest-velocity regions of the ejecta. As nebular-epoch spectroscopy sees in principle "through" the entire ejecta, which at that point have low optical depth at most frequencies, being able to model three different epochs allows us on the one hand to optimize our results as regards the abundance distribution as a function of radius, and on the other to identify any behaviour that is not simply the result of the evolution in time of the SN nebula, as determined by the progressive decrease in density. It should be noted here that, because of its artificial nature, W7 resembles the density and abundance distribution of delayed detonation models (e.g., Iwamoto et al. 1999) in the inner layers, such that it would be practically impossible to distinguish among them based on nebular spectra only. The main differences in the density distribution are found in the outer layers, reflecting the different energetics of the different models, while differences in composition are mainly at intermediate velocities, where again burning depends on energy. Therefore, based on the result of Ashall et al. (2014), who favour a relatively low-energy explosion, we use the W7 density model in our simulations (but retain the freedom to modify the abundance distribution somewhat). We also note that, as a matter of fact, delayed detonation mdoels are also artificial, as the transition from subsonic to supersonic burning is typically introduced arbitrarily.

We computed nebular spectra to match the observed spectra of SN 2014J using our NLTE nebular code. In SNe Ia most of the cooling occurs via forbidden lines of $\mathrm{Fe}$, but some permitted and semiforbidden transitions of lighter elements are also active coolants (e.g., the Ca II IR triplet and H\&K lines, Ca II] 7291,7324 A, Mg I] $4570 \AA$ ). A [Ni II] $7380 \AA$ Aemission line is also expected if stable $\mathrm{Ni}$ is produced in the explosion, and is weakly observed in the spectra of SN 2014J.

Our modelling strategy reflects our standard approach for nebular spectral series: we first reproduced the earliest spectrum, and then used the same parameters (density and composition as functions of radius/velocity) derived from that fit to model the later epochs. The assumption behind this approach is that the ejecta are transparent at all nebular epochs. If this is true, all spectra should be reproduced by changing the epoch only, which allows for expansion and more radioactive decay. This has usually been successful for time-series of spectra, of both SNe Ia and Ib/c (e.g., Mazzali et al. 2007c, 2015). Therefore, we start by discussing the fit of the spectrum taken 303 rest-frame days after $B$-band maximum. A rise time of 20 days (Ashall et al. 2014) has to be included in our models, so that the density can be properly rescaled. The three spectra have therefore epochs of 323, 374, and 435 rest-frame days after explosion, respectively. For all our models we assumed a distance modulus $\mu=27.86 \mathrm{mag}$, corresponding to a distance of 3.77 Mpc, following Ashall et al. (2014).

The spectrum at 323 days (Fig. 2) looks just like any other nebular spectrum of a normal SN Ia. The two strongest emission features (after correction for reddening) are two blends of Fe lines in the blue. The feature near $4800 \AA$ is dominated by [Fe III] (strongest lines at $4658,4701,4734 \AA$ ), while the one near $5200 \AA$ is composed mainly of [Fe II] lines (strongest ones at 5159, 5262, $5336 \AA$ ), but they both contain lines of both ions (e.g., [Fe II] lines at $4815,4890 \AA$, [Fe III] lines at 5270, $5412 \AA$ ). This means that it is not possible to tune the ratio of the strength of these two emission features, which is controlled by the ionization ratio and the populations of the upper levels of the emitting transitions and therefore depends on both temperature and density. Of these two factors, the former has a secondary effect though, as level populations are not in LTE. In this case, as was noted several times previously, the ionization degree is determined by the competition between heating and cooling, which depends on density and therefore - indirectly - also on composition. At the typical densities of SN Ia ejecta at an age of about 1 year, a gas composed exclusively of ${ }^{56} \mathrm{Ni}$ tends to be hot and highly ionised, as a significant part of it contributes to heating (via ${ }^{56} \mathrm{Co}$ decay). Therefore, Fe III is likely to prevail. However, this situation is actually quite rare in SNe Ia, and whenever it is observed it is in $\mathrm{SNe}$ that are peculiar spectroscopically, tend to be subluminous, and are best explained as sub-M(Ch) events (e.g., Mazzali \& Hachinger 2012; Mazzali et al. 2011). These SNe have in common a low density in the inner layers, which disfavours recombination, and so do the scenarios to which they have been associated.

The most natural way to cause the gas to achieve a lower degree of ionization appears to be adding stable iron. This effectively cools the ejecta, as stable $\mathrm{Fe}$ can be heated by radioactive decay products and then contributes to cooling via line emission, but is not itself the product of a process that produces any heating. Additionally, stable Fe cools via the same emission lines that Fe deriving 


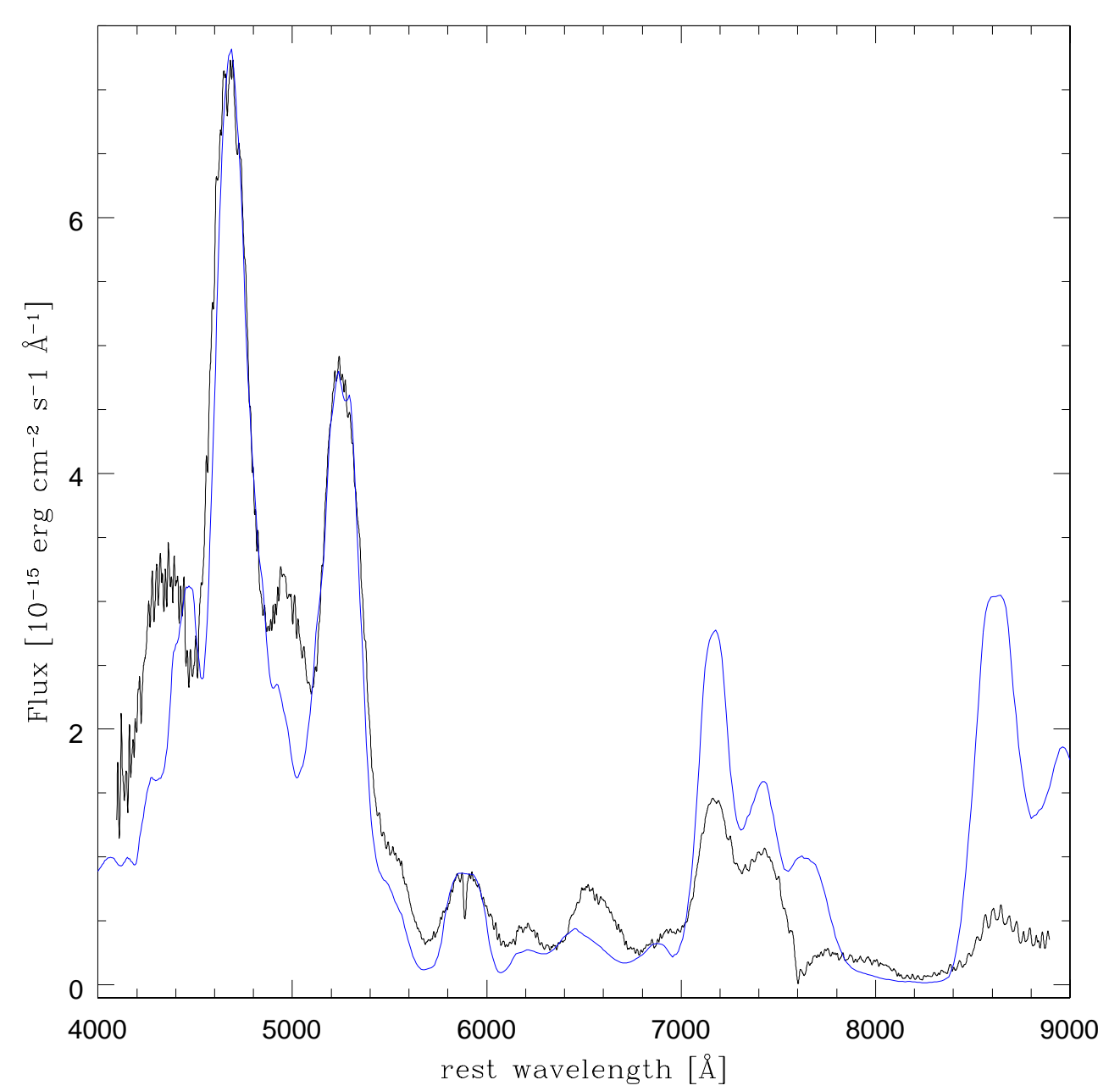

Figure 2. The synthetic nebular spectrum based on the 1D explosion model with stratified density and composition and optimised to match the features of SN 2014J at 323 rest-frame days after explosion (blue), compared to the observed SN spectrum after de-reddening and subtraction of the host spectrum (black).

from ${ }^{56}$ Co decay emits, thereby not causing unwanted spectral features. Introducing stable $\mathrm{Fe}$ in the Ni-rich ejecta indeed works well for all normal SNe Ia, and this is the case for SN 2014J, too. Calculations of explosive nucleosynthesis in near $\mathrm{M}(\mathrm{Ch}) \mathrm{SNe}$ Ia suggest that neutron-rich, stable isotopes of $\mathrm{Fe}$ and $\mathrm{Ni}$ (typically ${ }^{54} \mathrm{Fe}$ and ${ }^{58} \mathrm{Ni}$ ) are synthesized at the highest densities, in the centre of the WD (e.g., Iwamoto et al. 1999), but they are present with significant abundance (especially ${ }^{54} \mathrm{Fe}$ ) also in the ${ }^{56} \mathrm{Ni}$-dominated region. Sub-M(Ch) WDs do not reach the high densities necessary to synthesize stable $\mathrm{Fe}$ and $\mathrm{Ni}$, and therefore the presence or absence of stable $\mathrm{Fe}$ is an important element to discriminate between these two classes of models. The presence of stable Ni can be tested at late times via the emission of [ $\mathrm{Ni}$ II] 7380, as radioactive ${ }^{56} \mathrm{Ni}$ has almost completely decayed away at one year. On the other hand, emission from stable Fe just adds to the lines emitted by Fe produced by ${ }^{56} \mathrm{Co}$ decay, and as we cannot spectroscopically distinguish between different isotopes a direct measurement of the quantity of $\mathrm{Fe}$ synthesised as stable is not possible. A solution must be found whereby the balance between heating and cooling provides the correct strength and ratio of $[\mathrm{Fe} \mathrm{II}]$ and $[\mathrm{Fe} \mathrm{III}]$ lines, thereby matching both the ${ }^{56} \mathrm{Ni}$ production and the observed ionization within the volume comprised by the velocity of the Fe emission lines. A good balance between emission line intensity and the ratio of the iron ions that radiate was found in the case of SN 2014J for a ${ }^{56} \mathrm{Ni}$ mass of $\approx 0.46 \mathrm{M}_{\odot}$, located mostly at velocities between 3000 and $9000 \mathrm{~km} \mathrm{~s}^{-1}$, and a stable Fe mass of $\approx 0.24 \mathrm{M}_{\odot}$, located mostly at velocities between 1000 and $7000 \mathrm{~km} \mathrm{~s}^{-1}$. This is actually in line with typical explosion models of a Chandrasekhar-mass CO WD undergoing a delayed detonation (e.g., Iwamoto et al. 1999).

The results above are also in line with the expectations based on Mazzali et al. (2007a). The mass of ${ }^{56} \mathrm{Ni}$ is consistent with the decline rate of SN 2014J. The mass of stable Fe is also in line with other normal SNe Ia. It is required to fill the density structure of W7 as well as to keep the ionization sufficiently low. The fact that a good ionization balance is reached at the density of a $\mathrm{M}(\mathrm{Ch})$ model with a typical stable Fe content indeed simultaneously supports both the W7 density distribution and a $\mathrm{M}(\mathrm{Ch})$ explosion as the most likely scenario for SN 2014J. At 323 days Fe II and Fe III are present in similar amounts, with a ratio that depends on radius. The runs of density and $\mathrm{Fe}$ ionization as a function of depth are shown for this and the other models discussed in this paper in Fig- 


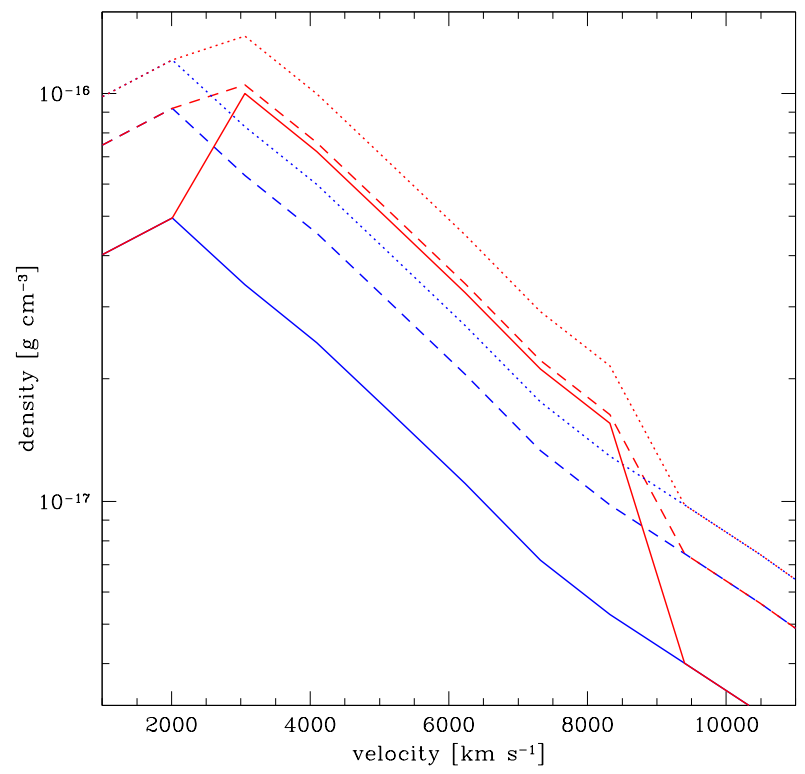

Figure 3. Density as a function of velocity in the Ni-rich ejecta for different epochs. The blue lines are the density of W7 at the times of the models, 323, 374 and 435 days from top to bottm, respectively, while the red lines show the density in the clumps.

ures 3 and 4, respectively. When the innermost parts of the density profile of $\mathrm{W} 7$ are filled with ${ }^{56} \mathrm{Ni}$ and stable $\mathrm{Fe}$ a reasonable ionization balance is reached, but best results are achieved if it is further assumed that the ${ }^{56} \mathrm{Ni}$-rich region is also clumped. Very moderate clumping is required (volume filling factors of $\sim 0.60$ ), similar to other normal SNe Ia, in regions where ${ }^{56} \mathrm{Ni}$ dominates the abundance. This leads to a slightly lower ionization of Fe. Confining all ${ }^{56} \mathrm{Ni}$ to lower velocities (higher densities) to favour recombination would actually change the width of the emission lines and yield unsuitable synthetic spectra (Mazzali et al. 2019).

The stable Ni mass, on the other hand, is quite small, $\approx$ $0.005 \mathrm{M}_{\odot}$, based on the intensity of the [Ni II] 7380 line. This result is in contrast with typical $\mathrm{M}(\mathrm{Ch})$ explosion models (e.g., Iwamoto et al. 1999), but is not unusual for normal SNe Ia, as we discuss below. Stable Ni has to be located mostly below $2000 \mathrm{~km}$ $\mathrm{s}^{-1}$ in order to avoid an excessively strong [Ni II] 7380 line. It is worth noting here that the [Ni II] line is not observed to have any shift with respect to its expected rest-frame position. Considering that SN 2014J is a marginal member of the low velocity gradient (LVG; Benetti et al. 2005) group of SNe Ia $(\dot{v}=-58.4 \pm 7.3 \mathrm{~km}$ $\mathrm{s}^{-1} \mathrm{~d}^{-1}$; Galbany et al. 2016), this is not in contradiction with a possible 3D picture of most $\mathrm{SNe}$ Ia as off-centre delayed detonation explosions (Maeda et al. 2010).

Regions in the ejecta lying outside a velocity of $\approx 7500 \mathrm{~km}$ $\mathrm{s}^{-1}$ were assumed to be characterised by the abundance distribution determined from the models of the early-time spectra in Ashall et al. (2014). These regions are dominated by intermediatemass elements (IME), in particular silicon and sulphur. They are separated from ${ }^{56} \mathrm{Ni}$ and therefore are not subject to much heating and do not radiate significantly. The ionization is therefore lower in those regions, as shown in Fig.4.

Having determined a likely solution for the $\mathrm{d} 323$ spectrum, we now move to the next epoch, $\mathrm{d} 374$. The expectation is that simply modifying the epoch should yield a good fit to the observed spec-

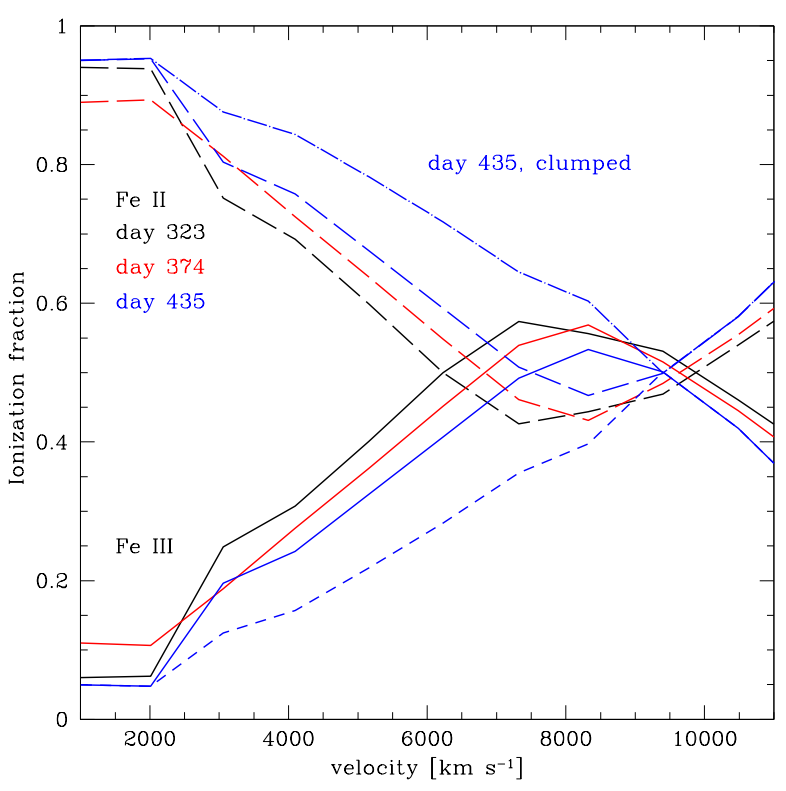

Figure 4. The ionization of $\mathrm{Fe}$ as a function of velocity in the ejecta for different epochs as computed by our model. The strongly clumped model for day 435 results in much lower ionization. The plot is limited to regions where energy deposition from gamma-rays and positrons is significant and leads to emission in Fe lines.

trum. This is indeed the case: Fig. 5 shows the observed spectrum of SN 2014J after dereddening and subtraction of the host galaxy spectrum together with the synthetic spectrum obtained using the same density and abundance distribution as on day 323, as well as the same clumping. The success of this test indicates that the model of the density and abundance distribution that we adopted is reasonably correct. There are a large number of SN Ia nebular spectra at epochs near 1 year, and the spectra of SN 2014J are similar to those of other normal SNe Ia.

Finally, we model the last spectrum of our series. This has an epoch of 435 days after explosion, and thus it samples an advanced epoch that had not been monitored in other SNe Ia, except for the spectrum of SN2011fe at 481 days after explosion published by Zhang et al. (2016) which is discussed above. It is characterised by the onset of the drop of the [Fe III] emission, although no strong $[\mathrm{Fe} \mathrm{I}]$ emission is yet visible.

If we use the same model as for the previous two epochs and just change the reference time we obtain the synthetic spectrum shown as a dashed line in Fig. 6. Although the synthetic spectrum does show a drop in [Fe III] emission, this is much less than what is actually observed. If no changes occur, the predicted degree of ionization in the model at this epoch is naturally lower than at earlier epochs because of the decreasing efficiency of gamma-ray deposition in the lower density nebula (see Fig. 4), but still high enough for significant [Fe III] emission to be present, which is in conflict with observations. A similar behaviour was noted at even much later epochs in the models of Fransson \& Jerkstrand (2015).

If we want to keep the radial density and abundance distribution that was successful at earlier phases and at the same time ensure a lower degree of ionzation, and thus a better match to the observed spectrum, the only change that we can make at this later epoch is to increase clumping. Fransson \& Jerkstrand (2015) also note in passing that clumping may improve the match between their 


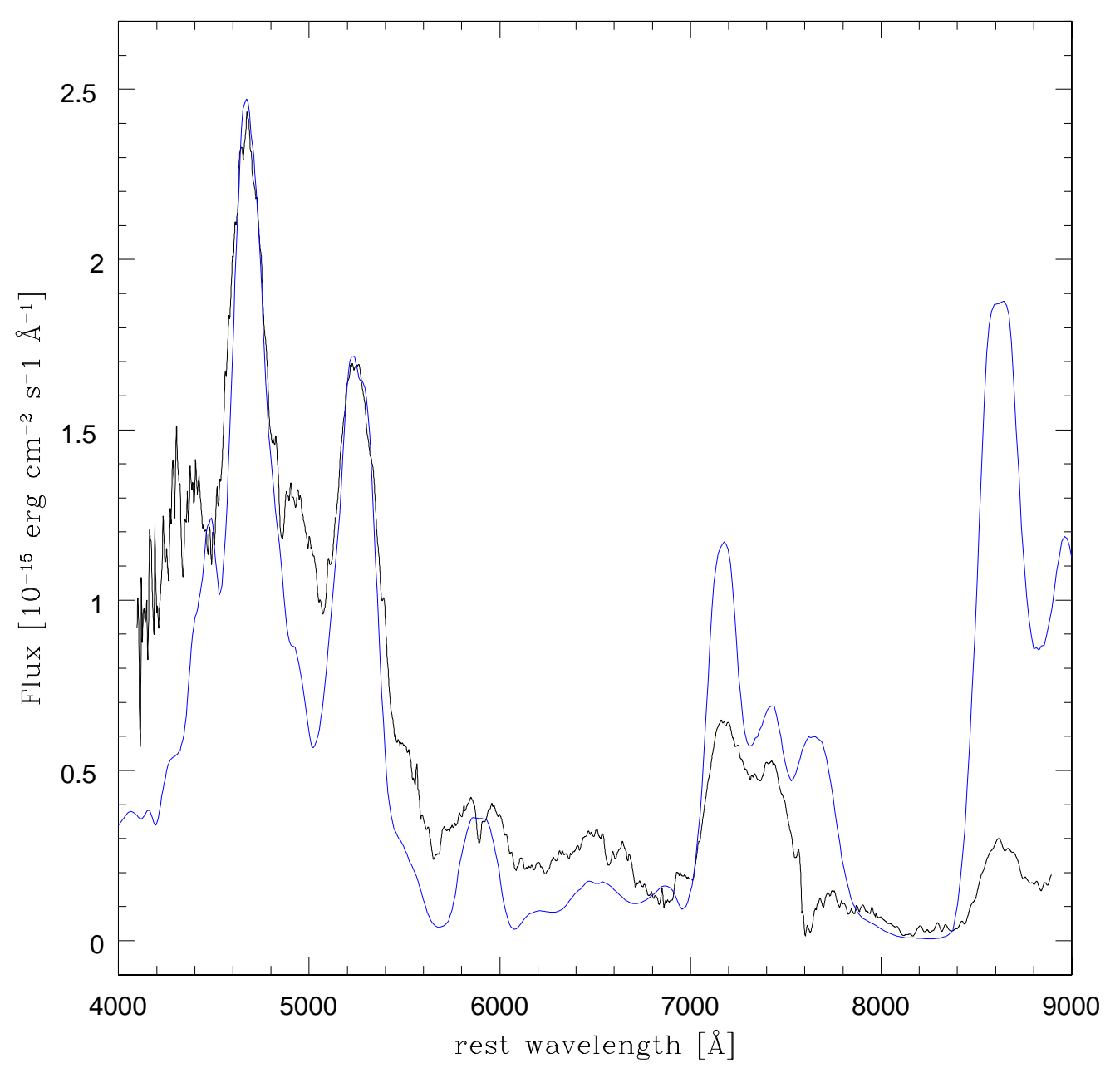

Figure 5. The synthetic nebular spectrum based on the 1D explosion model with stratified density and composition and optimised to match the features of SN 2014J at 374 rest-frame days after explosion (blue), compared to the observed SN spectrum after de-reddening and subtraction of the host spectrum (black).

synthetic spectra and the very late spectra of SN2011fe (they address much later epochs than we do here, when [Fe III] emission has completely disappeared).

Our approach to clumping affects in particular the regions that are rich in ${ }^{56} \mathrm{Ni}$, namely the inner ejecta. We do not separate different elements in the clumps, so that heating is largely unaffected (at these late epochs most energy deposition is due to positrons, which deposit locally as they typically have a short mean free path, and $\mathrm{Fe}$ coexists even at the microscopic level with ${ }^{56} \mathrm{Ni}$ and hence with the sites where positrons are created). Cooling takes place via the usual emission lines, but the higher densities in the clumps enhance recombination there, such that equilibrium is achieved at a lower ionization regime than in an unclumped or less clumped medium.

If we increase clumping significantly at the latest epoch a shift in ionization occurs and the observations can be reproduced. This can be achieved using a volume filling factor $f f=0.30$ in the ${ }^{56} \mathrm{Ni}$-rich ejecta at $t=435$ days. The corresponding synthetic spectrum is shown in Fig. 6 as a thick blue line. Increasing clumping implicitly means increasing the density in the regions of the ejecta that actively emit. The density as a function of velocity is shown in Fig. 3 for the three epochs, both without clumping and with the clumping that yields best fits to the Fe emission. Interestingly, the density at d435 when enhanced clumping is used is similar to the density of the clumped model at $\mathrm{d} 374$, so that the differences in ionization degree between the two epochs are due primarily to the lower rate of ionization. The enhanced clumping at $\mathrm{d} 435$ leads to significant changes in Fe ionization with respect to the model with the same degree of clumpping as at previous epochs, as can be seen in Fig. 4. For example, at $t=435$ days, the ratio $\mathrm{Fe}$ II/Fe III reaches values of 2-3 in the shells that mostly contribute to emission in the highly clumped model, while in the model with moderate clumping it is more like 1-2. With a lower ionization, [Fe III] emission is significantly reduced, and the synthetic spectrum reproduces the observations quite well. Although we had to increase clumping to suppress [Fe III] emission, the degree of clumpiness we used is not yet extreme, possibly indicating that the ejecta are undergoing a transition to a more clumpy structure. In the blue the spectrum has a lower signal-to-noise ratio. However, the difference between the clumped and unclumped models is clearly visible, and the more clumped model matches the data much better.

The abundances and clumping properties of the regions out- 


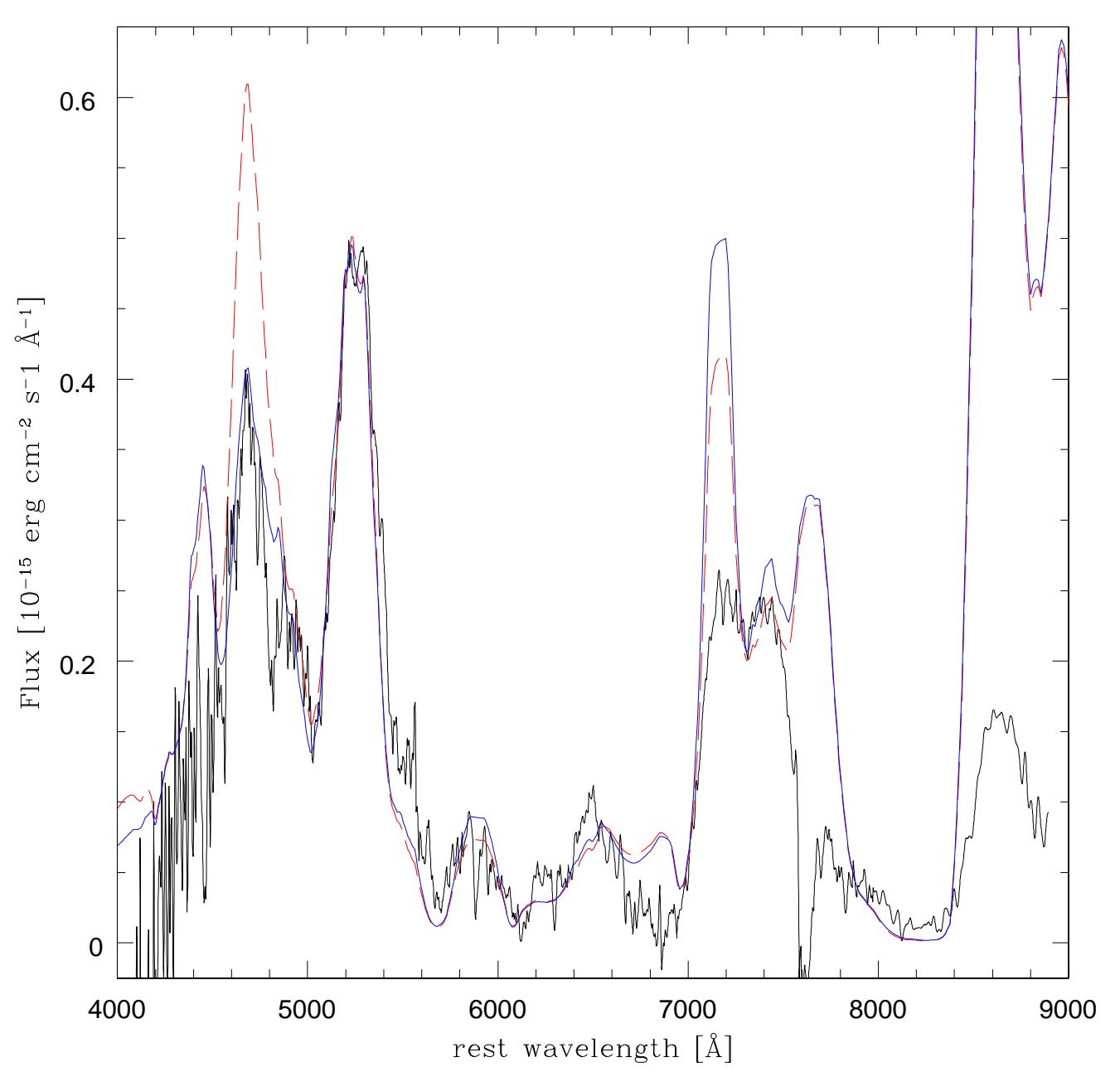

Figure 6. Synthetic nebular spectra based on the 1D explosion model with stratified density and composition, compared to the observed SN spectrum of SN 2014J at 435 rest-frame days after explosion after de-reddening and subtraction of the host spectrum (black). The dashed red line show the model with stratified density, composition and clumping as in previous epochs. The full blue line shows the 1D model with stratified density and composition as in previous epochs but enhanced clumping to suppress Fe III.

side those where ${ }^{56} \mathrm{Ni}$ dominates cannot be assessed using this method, as those regions are inefficiently heated by radioactive decay products at late epochs and are therefore largely passive. The abundance distribution in the ejecta of SN 2014J obtained by combining the early-time results of Ashall et al. (2014) and the nebular results obtained here is shown in Fig. 7. It is consistent with the results of several other normal SNe Ia, e.g., 2011fe (Mazzali et al. 2015) or 2004eo (Mazzali et al. 2008), although the production of $\mathrm{Si}$ and $\mathrm{S}$ appears to be somewhat suppressed. These results come from the analysis of the early time data in Ashall et al. (2014), and we do not discuss them again here.

\section{ASIDE: COULD EXTREME CLUMPING BE USED AT ALL EPOCHS?}

Given that we invoked clumping to suppress [Fe III] emission at very late times, one question that naturally arises is could clumping be more extreme than previously estimated also at earlier epochs during the nebular phase. More extreme clumping may be seen as a way to achieve a lower ionization, and therefore the correct balance of [Fe III] and [Fe II] emission, without having to resort to stable Fe to provide additional cooling. This seems to be the situation for example for $\mathrm{SNe} \mathrm{Ib} / \mathrm{c}$, where stable $\mathrm{Fe}$ is not supposed to be synthesized during the explosion and yet strong [Fe III] emission is seen at no point during the nebular phase. Filling factors of the order of 0.1 are required to suppress [Fe III] emission in $\mathrm{SNe} \mathrm{Ib/c}$ (e.g., Mazzali et al. 2007c), much more extreme than in the case of $\mathrm{SN}$ 2014J. However, SNe Ib/c are also thought to be highly aspherical (e.g., Maeda et al. 2006; Ashall et al. 2019), so clumping may also just mimic the effect of a non-smoothly spherical symmetric distribution of ${ }^{56} \mathrm{Ni}$ when modelling is performed in one dimension (e.g., Mazzali et al. 2015).

In the case of SN 2014J, we asked the question: if we increase clumping, eliminate stable $\mathrm{Fe}$ and thus just reduce the mass in the ejecta, can a consistent reproduction of the data be obtained? The three steps above are necessary in order to decrease ionization while avoiding cooling via unwanted emission lines of other elements that may be introduced when trying to conserve mass. What 


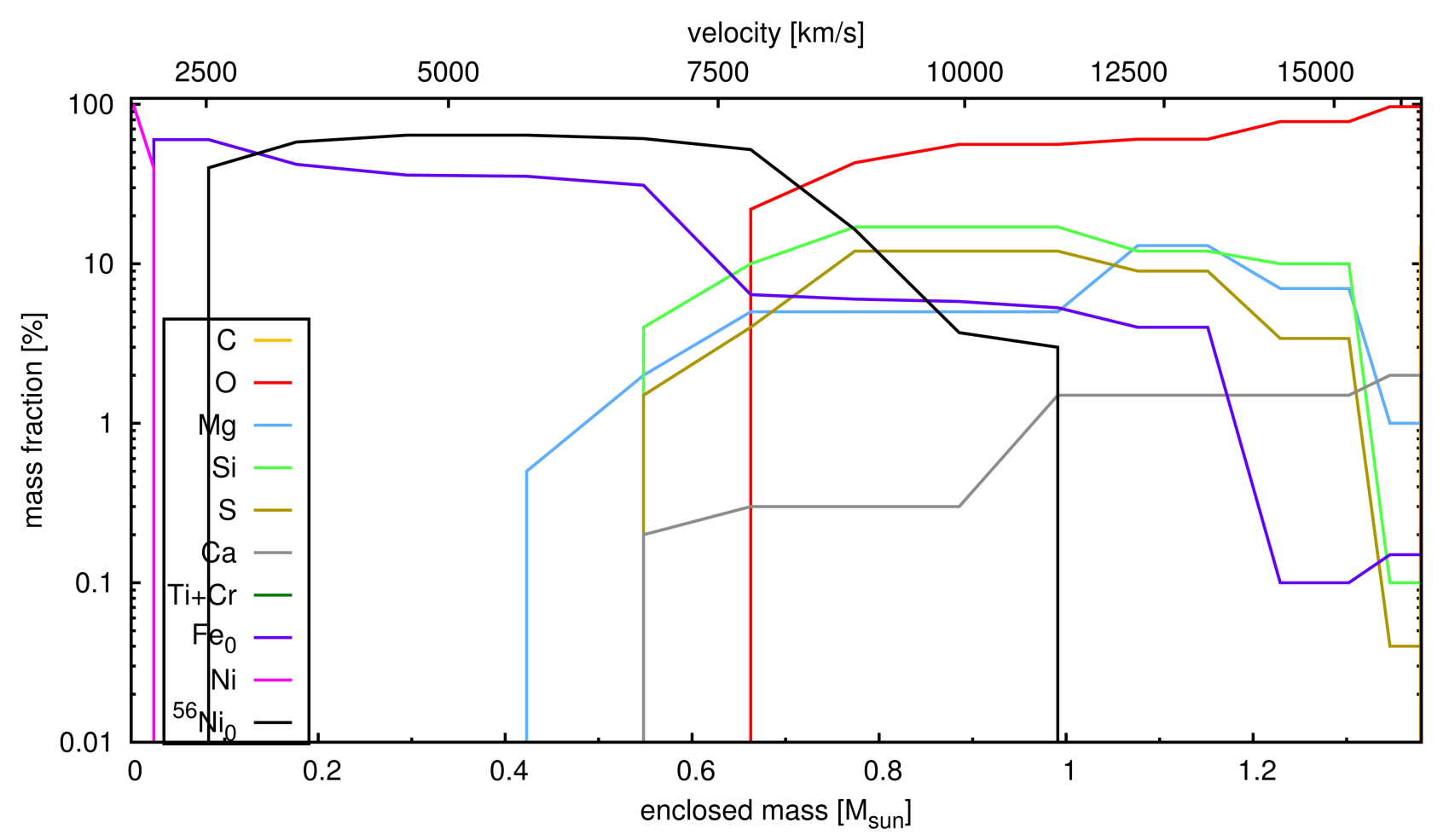

Figure 7. The abundance distribution in the ejecta of SN 2014J as determined combining the early-time results of Ashall et al. (2014) above $v=7500 \mathrm{~km}$ $\mathrm{s}^{-1}$ and the nebular result from the present paper at lower velocities.

we describe here amounts to constructing a model with the characteristics of a sub-M(Ch) explosion, a scenario that is favoured by some for the bulk of SNe Ia because searches for both surviving companions and the remains of accreted hydrogen, both of which are important features of the single degenerate scenario, have thus far failed to yield positive results. It shouod be noted, however, that possible ways to avoid those issues exist for both of these expected observations (e.g., Di Stefano, Voss \& Claeys 2011; Di Stefano \& Kilic 2012; Justham 2011).

We attempted to produce a reasonable synthetic nebular spectrum at $t=323$ days for an ejected mass $\mathrm{M}_{\mathrm{ej}} \sim 1.1 \mathrm{M}_{\odot}$. This value of $\mathrm{M}_{\mathrm{ej}}$ is in line with many sub-M(Ch) models (e.g., Sim et al. 2010). A resonable nebular spectrum could be obtained for a ${ }^{56} \mathrm{Ni}$ mass of $\sim 0.44 \mathrm{M}_{\odot}$ if we used quite an extreme clumping, i.e., a filling factor $f f=0.28$. The reduced ejected mass comes at the expense of the amount of material in the inner ejecta. In particular, the mass of stable $\mathrm{Fe}$ is only $0.03 \mathrm{M}_{\odot}$, while that of stable $\mathrm{Ni}$ remains very small, $\approx 0.002 \mathrm{M}_{\odot}$, because of the weakness of the [Ni II] 7380 line.

The synthetic spectrum thus obtained is shown in Fig.8. The quality of the fit is similar to that of the Chandrasekhar-mass model, which may be seen as a positive aspect for this solution. The only slight difference is that the Fe emission lines are somewhat narrower. A reduced core mass with practically unchanged outer layers is indeed a feature of sub-Chandrasekhar-mass models (see Mazzali et al. 2011). However, it also would probably imply a shorter diffusion time of photons at early times, and result in a narrower light curve at peak, as has indeed been observed for peculiar, subluminous SNe Ia (see Mazzali et al. 2011). It also must be noted that clumping is not currently a feature of sub-M(Ch) models either, let alone strong clumping.

However, this model is not stable, both in clumping and in ${ }^{56} \mathrm{Ni}$ mass. The strong clumping adopted for the model at $t=323$ days favours gamma-ray deposition, especially in the inner layers, such that if clumping remains constant in time then the later epochs are predicted to have higher emission flux than is actually observed. In order to fit the spectra the ${ }^{56} \mathrm{Ni}$ mass must be reduced as a function of time, which is unlikely to be an acceptable solution. At $t=374$ days the ${ }^{56} \mathrm{Ni}$ mass has to decrease to $0.40 \mathrm{M}_{\odot}$ for a reasonable fit, which is however not very good. A better fit can be achieved if clumping is slightly reduced, to $f f=0.32$ (see Fig.9). The stable Fe mass is now larger, $0.06 \mathrm{M}_{\odot}$, while the stable $\mathrm{Ni}$ mass is still $0.002 \mathrm{M}_{\odot}$. At both of these epochs, in addition to the problems mentioned above, the removal of mass makes it difficult to produce broad enough $\mathrm{Fe}$ emission features, even though the inner regions (up to $v \approx 7000 \mathrm{~km} \mathrm{~s}^{-1}$ are now dominated by ${ }^{56} \mathrm{Ni}$. This is because of the missing Fe emission and the reduced mass. 


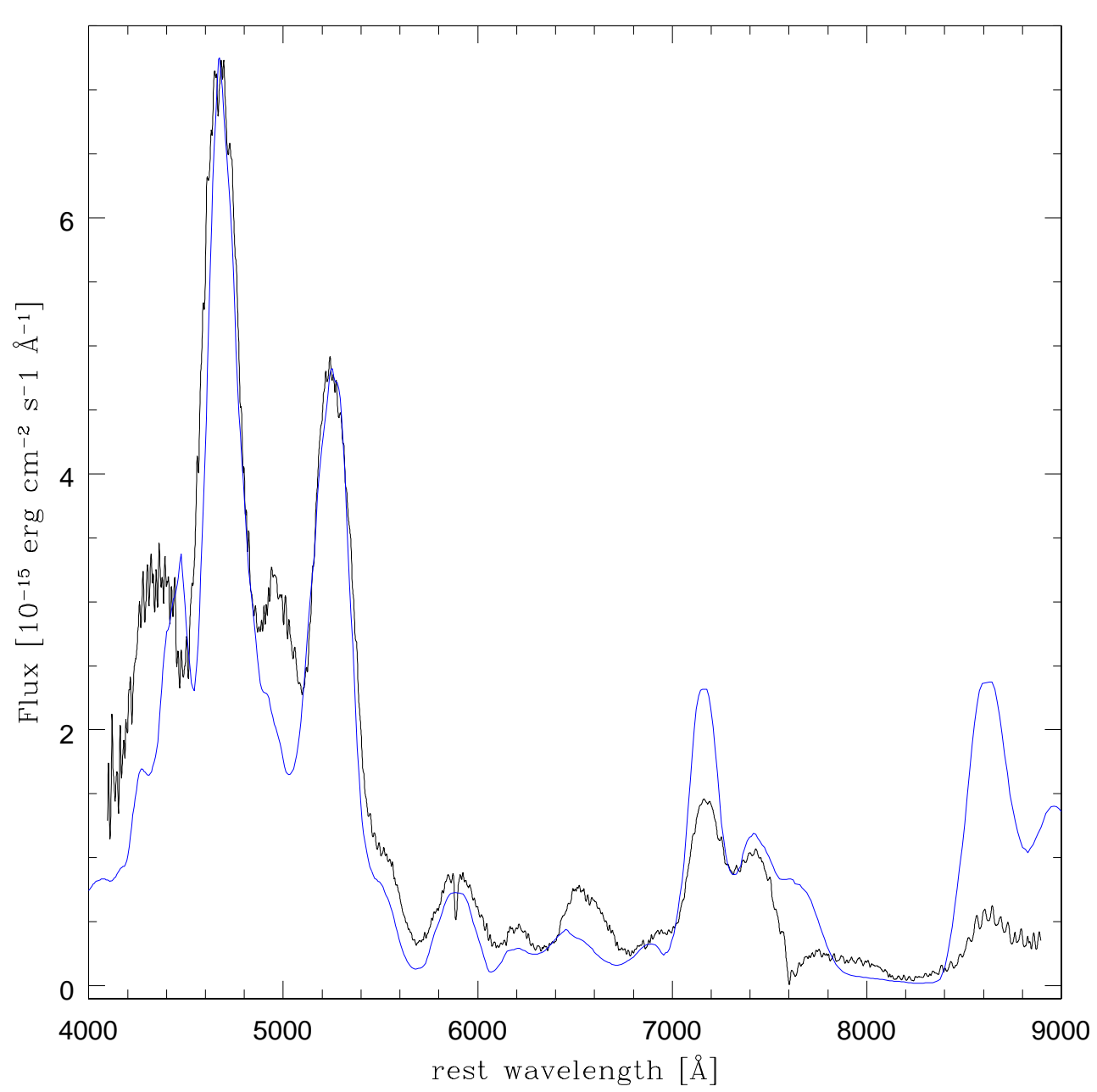

Figure 8. The synthetic nebular spectrum based on the 1D explosion model with ejected mass $1.1 \mathrm{M}_{\odot}$, stratified density and composition, compared to the observed SN spectrum at 323 rest-frame days after explosion after de-reddening and subtraction of the host spectrum (black).

At $t=435$ days, however, in order to obtain a good fit, the ${ }^{56} \mathrm{Ni}$ mass must increase again somewhat (now $0.42 \mathrm{M}_{\odot}$, at the expense of stable $\mathrm{Fe}$, now $0.04 \mathrm{M}_{\odot}$ ) but clumping must again increase significantly, now to $f f=0.18$ (see Fig.10). So, irrespective of the mass adopted, strong clumping is needed at the late epoch.

Is the sub-M(Ch) scenario a viable solution? The somewhat poorer fits to the data on the one hand, together with the unphysical requirement of a time-dependent ${ }^{56} \mathrm{Ni}$ on the other, make us favour the Chandrasekhar-mass model with increasing clumping at the latest epoch, as fewer time-dependent modifications are necessary in this case. Additionally, a reduced mass is likely to lead to a more rapidly evolving light curve near peak. The kinetic energy of the sub-M(Ch) model was not greatly reduced, and mass was removed only from the deepest, lowest-velocity layers, both in order to test the nebular spectra and to keep the density/abundance structure that was successfully used to fit the early-time spectra (Ashall et al. 2014).

\section{DISCUSSION}

Our results indicate that the increase of clumping in the ejecta of SN 2014J at epochs past one year after explosion may have been responsible for the decrease in ionization which in turn is reflected in the evolving spectral properties, with a drop in [Fe III] emission.

The presence of significant clumping at the time of the latest of our three nebular spectra of SN 2014J suggests that the ejecta evolve morphologically, making a transition from a smoother regime which applies earlier on to a more clumpy state. In SN 2014J this transition appears to start about one year after the explosion. It may continue into later times, leading to even more extreme clumping, but unfortunately later data are not available for SN 2014J. Evidence from SN 2011fe is that eventually [Fe III] lines disappear and are replaced by [Fe I] lines, which suggests that clumping may be active in that $\mathrm{SN}$, too, as suggested by Fransson \& Jerkstrand (2015). Evidence for increased clumping in SN 2014J comes from the spectrum at 435 days. Previous work on nebular spectra of SNe Ia had been limited to spectra with epochs barely exceeding one year (e.g., Mazzali et al. 2007a), and such a shift in ionization was not seen in any of the normal SNe Ia that were analysed in that work. In the future it will be important to 


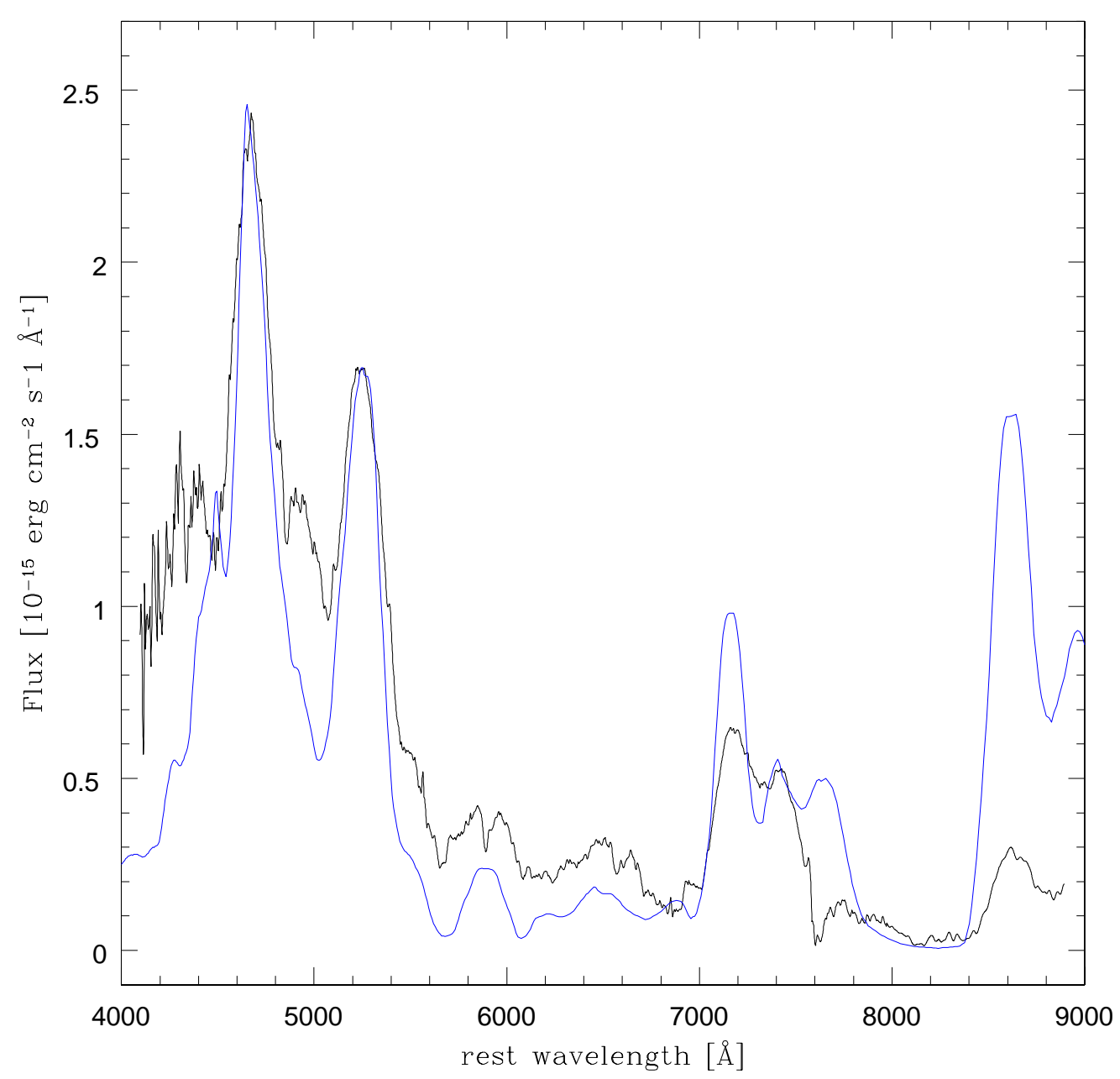

Figure 9. The synthetic nebular spectrum based on the 1D explosion model with ejected mass $1.1 \mathrm{M}_{\odot}$, stratified density and composition, compared to the observed SN spectrum at 374 rest-frame days after explosion after de-reddening and subtraction of the host spectrum (black).

make every effort to follow SNe into the nebular phase, and well after one year whenever possible, in order to establish whether the behaviour of SN 2014J (and SN 2011fe) is actually common to the bulk of (at least normal) SNe Ia.

Our description of the clumpy properties of SN 2014J is a very simple one. It is not easy to extract such information from the spectrum. Our one-dimensional modelling has clear limitations, but if one resorted to 3D modelling the number of free parameters (clump size, density, composition, distribution as well as any underlying deviation from spherical symmetry in the explosion model) would be so large that finding a solution may be quite hard, and multiple solutions may be found. As we mentioned above, current SN Ia explosion models do not predict the development of clumps, but this is a feature that future models may have. For the SN IIP-peculiar 1987A Jerkstrand et al. (2011) used a multi-dimensional model to inform their simulations. No available SN Ia model predicts either clumpy ejecta or the development of clumps with time, so for the time being our approach appears at least to bring out the nature of the physical process at play, although not its details.

An interesting scenario is suggested looking at Fig. 3: the model with enhanced clumping at $\mathrm{d} 435$ has very similar densities as the moderately clumped model at $\mathrm{d} 374$. This suggests that clumps may form, or be present in the ejecta, about one year after the explosion, and that they do not expand, behaving more like shrapnel than small gas clouds. This may indeed be consistent with the observed properties of SNRs.

A possible cause for the onset of clumping could be the development of magnetic fields caused perhaps by the relative motion of charged particles such as the ions and electrons in the ejecta. The young SN nebula is significantly ionized, reaching electron densities up to $\sim 10^{6} \mathrm{~cm}^{-3}$ in the inner ejecta at 435 days in the model with enhanced clumping, so this is a possibility, especially in the presence of velocity offsets between different species. The actual details are likely to be quite complex, and are certainly well beyond the possibilities of our spectrum synthesis approach and the scope of this paper. Magneto-hydrodynamic calculations would be required, and those are encouraged.

The small mass of stable $\mathrm{Ni}$ is in contrast with typical Chandrasekhar-mass model predictions. Such models (e.g., Iwamoto et al. 1999) typically foresee a ratio of stable Ni to stable Fe of $\sim 1$. In all Chandrasekhar-mass models we have computed (e.g., Mazzali et al. 2007a, 2015) this ratio is actually much smaller 


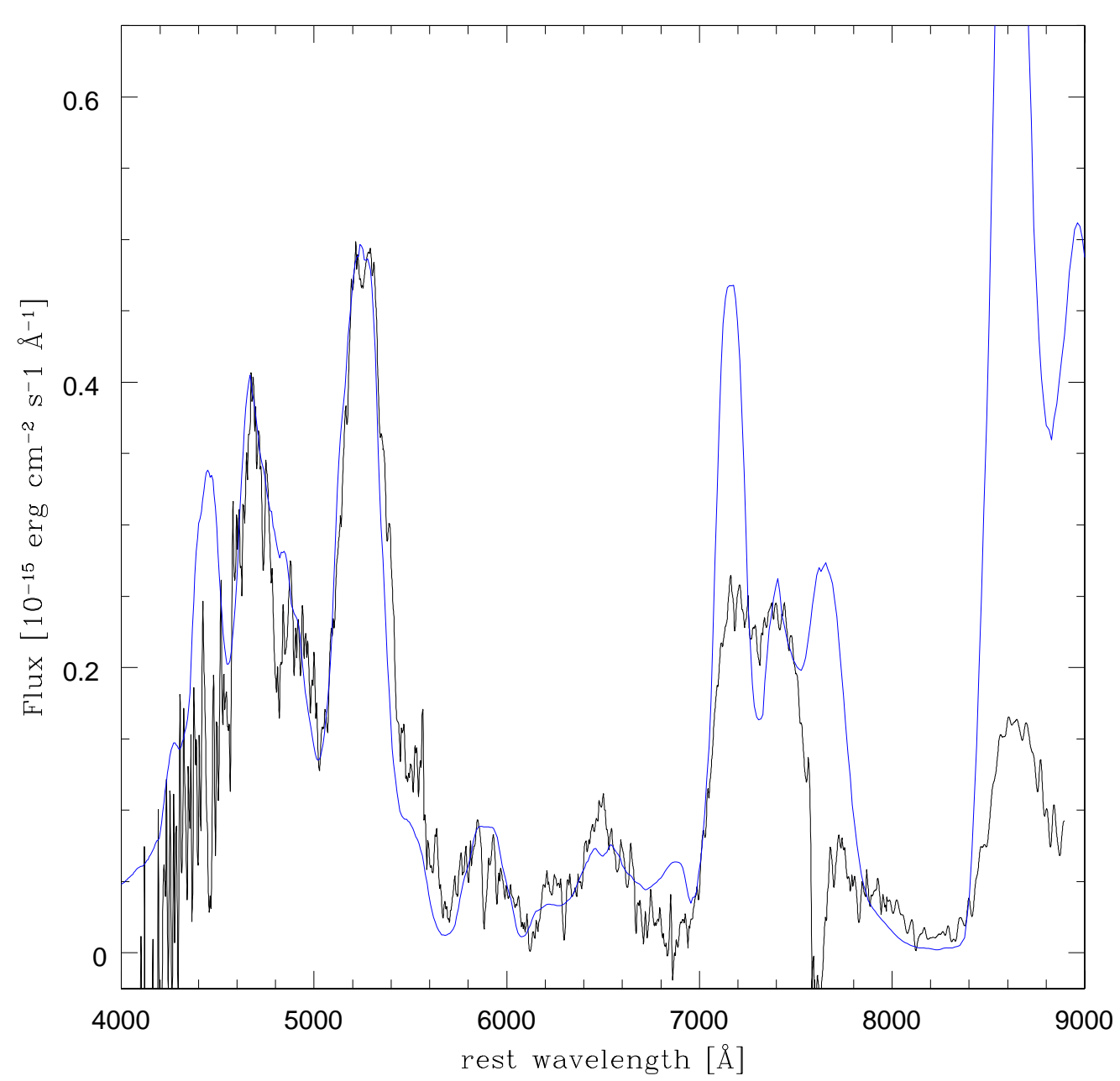

Figure 10. The synthetic nebular spectrum based on the $1 \mathrm{D}$ explosion model with ejected mass $1.1 \mathrm{M}_{\odot}$, stratified density and composition, compared to the observed SN spectrum at 435 rest-frame days after explosion after de-reddening and subtraction of the host spectrum (black).

Table 2. Properties of one-zone models

\begin{tabular}{ccccccc}
$\begin{array}{c}\text { Epoch } \\
(\text { days })\end{array}$ & $\begin{array}{c}\mathrm{M}\left({ }^{56} \mathrm{Ni}\right) \\
{\left[\mathrm{M}_{\odot}\right]}\end{array}$ & $\begin{array}{c}\mathrm{M}(\mathrm{Fe}) \\
{\left[\mathrm{M}_{\odot}\right]}\end{array}$ & $\begin{array}{c}\mathrm{M}(\text { stable } \mathrm{Ni}) \\
{\left[\mathrm{M}_{\odot}\right]}\end{array}$ & $\begin{array}{c}\mathrm{M}_{\text {tot }} \\
{\left[\mathrm{M}_{\odot}\right]}\end{array}$ & $\begin{array}{c}\text { velocity } \\
{\left[\mathrm{km} \mathrm{s}^{-1}\right]}\end{array}$ & ff \\
\hline 323 & 0.66 & 0.04 & 0.01 & 0.76 & 7700 & 0.50 \\
374 & 0.67 & 0.03 & 0.02 & 0.77 & 9000 & 0.50 \\
435 & 0.73 & 0.03 & 0.02 & 0.83 & 8000 & 0.50 \\
\hline
\end{tabular}

than 1 . The small Ni mass stems from the weakness of the only observed Ni line in the nebular spectrum, [Ni II] 7380, which we can fit with a stable $\mathrm{Ni}$ mass of $\approx 0.005 \mathrm{M}_{\odot}$. A low $\mathrm{Ni} / \mathrm{Fe}$ ratio was also recently obtained by Flörs et al. (2020), who claim that it suggests that the bulk of SNe Ia are sub-M(Ch) explosions. However, sub$\mathrm{M}(\mathrm{Ch})$ explosions also produce very little stable Fe directly: most stable Fe is just the result of the decay of ${ }^{56} \mathrm{Ni}$. Flörs et al. (2020) use one-zone models to determine the final ratio of $\mathrm{Fe}$ (including both stable Fe directly synthesized and Fe produced by ${ }^{56} \mathrm{Ni}$ decay). We computed one-zone models for the three nebular spectra of SN 2014J presented here in order to test the results. We could obtain reasonable fits for the three spectra using the parameters listed in Table 2. The fits were driven by the effort to match the strength and width of the strongest emission lines, which are complex blends, as we remarked above. One-zone models are very useful to determine the actual extent of the effectively emitting zone, as they take line blending into account. Just like Flörs et al. (2020), we find that the stable Ni mass is small, and in addition we also do not require much stable Fe. However, in a one-zone models the real conditions in the ejecta are not well reproduced. In particular, the density is constant within the emitting sphere, which is far from reality. In our one-zone models we adopted a filling factor of 0.5 as a proxy for the density distribution, otherwise we could not achieve a balance between the emitting flux, the width of the lines, and the ionization balance. In our 1D models we find a small mass of stable Ni because the single visible Ni line, [Ni II] 7380, is 
weak, but this is a typical feature of all SNe Ia. However, the mass of directly made stable Fe is large, and the total mass of stable Fegroup elements produced is actually consistent with the prediction of Chandrasekhar-mass explosion models. We also find that stable Ni tends to be at lower velocities than directly made stable Fe as well as ${ }^{56} \mathrm{Ni}$, which is again consistent with Chandrasekhar-mass models. One possibility is that differences between the central density estimated in models and their real value may lead to a different nucleosysnthesis than predicted.

\section{CONCLUSIONS}

We have modelled three nebular spectra of SN 2014J. We find that a good match to the observed spectra can be obtained if SN 2014J was a Chandrasekhar-mass explosion. The SN produced $\sim 0.45 \mathrm{M}_{\odot}$ of ${ }^{56} \mathrm{Ni}$. It also produced some $0.3 \mathrm{M}_{\odot}$ of stable $\mathrm{Fe}$, and a small amount $\left(\sim 0.005 \mathrm{M}_{\odot}\right)$ of stable Ni. The mass of ${ }^{56} \mathrm{Ni}$ that we obtain is in line with expectations for a SN Ia with the luminosity of SN 2014J. The mass of stable Ni is small compared to the predictions of Chandrasekhar-mass explosion models, but the mass of stable $\mathrm{Fe}$ is larger than in those models, such that the combined mass of stable Fe-group material produced directly is in line with model predictions. A low mass of stable $\mathrm{Ni}$ is found in all SN Ia whose nebular spectra we have modelled, including those compatible with the Chandrasekhar-mass. This suggests that SN 2014J was a Chandrasekhar-mass explosion, and that the detailed nucleosynthetic predictions of some explosion models are not correct.

In order to match the sharp decrease in emission intensity of the Fe III-dominated feature near $4800 \AA$ at the latest epoch, 435 days after explosion, we had to increase clumping at that epoch. Although our approach to clumping is very simplistic, namely we assume that all material is within the clumps and completely mixed according to the radial distribution of abundances, our results are very suggestive. Increase in clumping may be a general behaviour in SNe Ia. In the only case when extremely late spectra have been obtained, SN 2011fe, [Fe III] emission is seen to disappear almost completely at epochs of 2 years and later, and is replaced by [Fe I] lines. Interestingly, the increased clumping implies roughly constant density within the clumps between $\mathrm{d} 374$ and d435, suggesting that clumps at that point start behaving like shrapnel. Clumping may be caused by the development of magnetic fields in the SN ejecta, and it may mark the beginning of the transition to a SNR.

Acknowledgements. The authors thank TUBITAK, KFU, IKI, and AST for partial support in using RTT150 (Russian-Turkish 1.5-m telescope in Antalya). This work was partially funded by the Russian Government Program of Competitive Growth of Kazan Federal University.

\section{REFERENCES}

Amanullah, R., et al. 2014, ApJ, 788, L21

Ashall, C., Mazzali, P., Bersier, D., Hachinger, S., Phillips, M., Percival, S., James, P., \& Maguire, K. 2014, MNRAS, 445, 4427

Ashall, C., Mazzali, P. A., Pian, E., \& James, P. A. 2016, MNRAS, 463, 1891

Ashall, C., et al. 2018, MNRAS, 477, 153

Ashall, C., et al. 2019, MNRAS, 487, 5824

Aslan, Z., et al., 2001, Astron. Lett., 27, 398

Axelrod, T. S. 1980, Ph.D. Thesis, Univ. of California, Santa Cruz.

Benetti, S., et al. 2016, ApJ, 623, 1011
Bulla, M., Sim, S. A., Pakmor, R., Kromer, M., Taubenberger, S., Röpke, F. K., Hillebrandt, W., \& Seitenzahl, I. R. 2016, MNRAS, 455, 1060

Cappellaro, E., Mazzali, P. A., Benetti, S., Danziger, I. J., Turatto, M., della Valle, M., \& Patat, F. 1997, A\&A, 328, 203

Dhawan S., et al. 2018, A\&A, 619, A102

Diamond T. R., et al. 2018, ApJ, 861, 119

Di Stefano R., Voss R., \& Claeys J. S. W., 2011, ApJL, 738, L1

Di Stefano R., \& Kilic, M., 2012, ApJ, 759, 56

Ferrand, G., Warren, D. C., Ono, M., Nagataki, S., Röpke, F. K., \& Seitenzahl, I. R. 2019, ApJ, 877, 136

Flörs, A., et al. 2020, MNRAS, 491, 2902

Foley, R. J., et al. 2014, MNRAS, 443, 2887

Fransson, C., \& Jerkstrand, A. 2015, ApJ, 814, L2

Graham, M. L., et al. 2015, MNRAS, 454, 1948

Galbany, L., et al. 2016, MNRAS, 457, 525

Iben, I., Jr., \& Tutukov, A. V. 1984, ApJS, 54, 335

Iwamoto, K., Brachwitz, F., Nomoto, K., Kishimoto, N., Umeda, H., Hix, W. R., \& Thielemann, F. 1999, ApJS, 125, 439

Jerkstrand, A., Fransson, C., \& Kozma, C. 2011, A\&A, 530, A45

Justham, S. 2011, ApJL, 730, L34

Kashi, A., \& Soker, N. 2011, MNRAS, 417, 1466

Kozma, C. \& Fransson. 1998, ApJ, 497, 431

Kushnir, D., Katz, B., Dong, S., Livne, E., \& Fernández, R. 2013, ApJ, 778, L37

Livio, M. \& Mazzali, P. 2018, Phys. Rep., 736, 1

Livio, M. \& Riess, A. G. 2003, ApJ, 594, L93

Livne, E. \& Arnett, D. 1995, ApJ, 452, 62

Maeda, K., Mazzali, P. A., \& Nomoto, K. 2006, ApJ, 645, 1331

Maeda, K., et al. 2010, Nature, 466, 82

Mazzali, P. A., Cappellaro, E., Danziger, I. J., Turatto, M., \& Benetti, S. 1998, ApJ, 499, L49

Mazzali, P. A., \& Hachinger, S. 2012, MNRAS, 424, 2926

Mazzali, P. A., Maurer, I., Stritzinger, M., Taubenberger, S., Benetti, S., \& Hachinger, S. 2011, MNRAS, 416, 881

Mazzali, P. A., Moriya, T. J., Tanaka, M., Woosley, S. E. 2011, MNRAS, 484,3451

Mazzali, P. A., Nomoto, K., Cappellaro, E., Nakamura, T., Umeda, H., Iwamoto, K. 2001a, ApJ, 547, 988

Mazzali P. A., Nomoto K., Patat F., Maeda K., 2001, ApJ, 559, 1047

Mazzali, P. A., Röpke, F. K., Benetti, S., \& Hillebrandt, W. 2007a, Science, 315,825

Mazzali, P. A., Sauer, D. N., Pastorello, A., Benetti, S., \& Hillebrandt, W. 2008, MNRAS, 386, 1897

Mazzali, P. A., et al. 2007b, ApJ, 661, 892

Mazzali, P. A., et al. 2007c, ApJ, 670, 592

Mazzali, P. A., et al. 2015, MNRAS, 450, 2631

Mazzali P. A., Ashall, C., Pian, E., Stritzinger, M. D., Gall, C., Phillips, M. M., Höflich, P., \& Hsiao, E. 2018, MNRAS, 476, 2905

Nomoto K., Thielemann F., Yokoi K. 1984, ApJ, 286, 644

Nugent, et al. 2011, Nature, 480, 344

Patnaude, D. J., Lee, S.-H., Slane, P. O., Badenes, C., Nagataki, S., Ellison, D. C., \& Milisavljevic, D. 2017, ApJ, 849, 109

Perlmutter S., et al. 1999, ApJ, 517, 565

Piersanti L., Gagliardi S., Iben I., Tornambé A. 2003, ApJ, 598, 1229

Pinto, P. A., \& Eastman, R. G. 2000, ApJ, 530, 757

Phillips, M. M. 1993, ApJ, 413, L105

Piascik A. S., Steele I. A., Bates S. D., Mottram C. J., Smith R. J., Barnsley R. M., Bolton B. 2014, SPIE, 9147, 91478H

Riess A. G., et al. 1998, AJ, 116, 1009

Röpke, F. K., et al. 2012, ApJ, 750, L19

Rosswog, S., Kasen, D., Guillochon, J., \& Ramirez-Ruiz, E. 2009, ApJ, 705, L128

Ruiz-Lapuente, P. \& Lucy, L.B. 1992, ApJ, 400, 127

Sim, S. A., Röpke, F. K., Hillebrandt, W., Kromer, M., Pakmor, R., Fink, M., Ruiter, A. J., Seitenzahl, I. R. 2010, ApJ, 714, L52

Shen K. J., Moore K. 2014, ApJ, 797, 46

Srivastav S., Ninan J. P., Kumar B., Anupama G. C., Sahu D. K., Ojha D. K., Prabhu T. P., 2016, MNRAS, 457, 1000 
Steele, I. A., et al. 2004, SPIE, 5489, 679

Taubenberger, S., et al. 2015, MNRAS, 448, L48

Webbink, R. F. 1984, ApJ, 277, 355

Whelan, J., \& Iben, I., Jr. 1973, ApJ, 186, 1007

Yang Y., et al. 2018, ApJ, 852, 89

Zhang K., et al. 2016, ApJ, 820, 67 